\title{
DIMS Experiment for Dark Matter and Interstellar Meteoroid Study
}

\author{
S. Abe, ${ }^{a}$ M. Arahori, ${ }^{b}$ D. Barghini, ${ }^{c, g}$ M. Bertaina, ${ }^{c}$ M. Casolino, ${ }^{e, f}$ A. Cellino, ${ }^{g}$ C. \\ Covault, ${ }^{r}$ T. Ebisuzaki, ${ }^{e}$ M. Endo, ${ }^{a}$ M. Fujioka, ${ }^{j}$ Y. Fujiwara, ${ }^{h}$ D. Gardiol, ${ }^{g}$ M. \\ Hajdukova, ${ }^{i}$ M. Hasegawa, ${ }^{a}$ R. Ide,${ }^{b}$ Y. Iwami, ${ }^{j}$ F. Kajino, ${ }^{b, *}$ M. Kasztelan, ${ }^{q}$ K. \\ Kikuchi, ${ }^{a}$ S.-W. Kim, ${ }^{k}$ M. Kojro, ${ }^{l}$ J.N. Matthews, ${ }^{m}$ K. Nadamoto, ${ }^{b}$ I.H. Park, ${ }^{n}$ L.W. \\ Piotrowski, ${ }^{o}$ H. Sagawa, ${ }^{p}$ K. Shinozaki, ${ }^{q}$ D. Shinto, ${ }^{j}$ J.S. Sidhu, ${ }^{r}$ G. Starkman, ${ }^{r}$ S. \\ Tada, $^{b}$ Y. Takizawa, ${ }^{e}$ Y. Tameda, ${ }^{j}$ S. Valenti ${ }^{c}$ and M. Vrabel ${ }^{q}$ \\ ${ }^{a}$ Department of Aerospace Engineering, Nihon University, Japan \\ ${ }^{b}$ Department of Physics, Konan University, Japan \\ ${ }^{c}$ Department of Physics, University of Turin, Italy \\ ${ }^{d}$ National Institute for Nuclear Physics (INFN) - Turin, Italy \\ ${ }^{e}$ RIKEN (Institute of Physical and Chemical Research), Japan \\ ${ }^{f}$ National Institute for Nuclear Physics (INFN) - Rome Tor Vergata, Italy \\ ${ }^{g}$ Observatory of Turin, National Institute for Astrophysics (INAF), Italy \\ ${ }^{h}$ Nippon Meteor Society (NMS), Japan \\ ${ }^{i}$ Astronomical Institute, Slovak Academy of Sciences, Slovakia \\ ${ }^{j}$ Department of Engineering and Science, Osaka Electro-Communication University (OECU), Japan \\ ${ }^{k}$ Korea Astronomy and Space Science Institute (KASI), Republic of Korea \\ ${ }^{l}$ Faculty of Physics and Applied Informatics, University of Lodz, Poland \\ ${ }^{m}$ Department of Physics and Astronomy, University of Utah, USA \\ ${ }^{n}$ Department of Physics, Sungkyunkwan University, Republic of Korea \\ ${ }^{o}$ Department of Physics, University of Warsaw, Poland \\ ${ }^{p}$ Institute for Cosmic Ray Research, University of Tokyo, Japan \\ ${ }^{q}$ National Centre for Nuclear Research (NCBJ), Poland \\ ${ }^{r}$ Department of Physics, Case Western Reserve University, USA \\ E-mail: kajino@konan-u.ac.jp
}

\footnotetext{
${ }^{*}$ Presenter
} 
DIMS (Dark matter and Interstellar Meteoroid Study) is a new experiment aiming to search for macroscopic dark matters and interstellar meteoroids. Nuclearites are nuggets of stable strange quark matter(SQM), neutral in charge and hypothetical super-heavy macroscopic particles (macros), and may be important components of the dark matter in our Universe. Nuclearites of galactic origins would have an expected typical velocity of about $250 \mathrm{~km} / \mathrm{s}$ in galactic frame, whereas in the case of a head-on collision between interstellar meteoroids with a velocity that exceeds the escape velocity of the solar system and the Earth orbiting the Sun, the geocentric velocities will be larger than $72 \mathrm{~km} / \mathrm{s}$. We study the possibility to search for such fast-moving particles by using very high-sensitivity CMOS cameras with a wide field of view. Based on observational data of meteor events using such stereo camera systems at some locations, we estimate the observable mass ranges for the moving nuclearites and the interstellar meteoroids. Observable flux limits are also estimated for these mass ranges. We designed the DIMS experiment to search for such particles. In its first stage, the DIMS system consists of 4 high-sensitivity CMOS camera stations with a wide field of view. The system is going to be constructed at the Telescope Array cosmic-ray-experiment site in Utah, USA. Details of the project science, plans and present status with test results are described in this paper.

$37^{\text {th }}$ International Cosmic Ray Conference (ICRC 2021)

July 12th - 23rd, 2021

Online - Berlin, Germany 


\section{Introduction}

\subsection{Macroscopic Dark Matter}

Traditionally, the leading candidates for dark matter (DM) possess very small cross-sections. However, as pointed out in [1], it would also be possible for DM to be constituted of particles characterized by high masses and cross-sectional areas, with low number density. This broad class of macroscopic DM candidates goes under the name of macros, and its members are supposed to interact with regular matter through their geometrical cross-section (see [1] and reference therein). Several models of macros are either directly or indirectly based on the hypothesis that strange quark matter (SQM) composed of $\mathrm{u}, \mathrm{d}$ and s quarks may be stabler than ordinary nuclear matter. In 1984 E. Witten [2] proposed that lumps of SQM might be present in our Universe as a result of the early Universe first-order phase transition from the state of quark-gluon plasma to ordinary baryonic matter. While this scenario is absolutely fascinating, there is no proof that such objects were produced in the early Universe. Nevertheless, there are other conditions that would allow the Universe to be populated with lumps of SQM. For instance, the high pressures created in the cores of neutron stars would create perfect conditions for developing SQM. Moreover, if we assume SQM to be stabler than ordinary matter, then it might be possible for neutron stars to convert most of their baryonic content into strange matter, except for a thin outer layer made out of ordinary nuclei [3]. Lastly, astrophysical events such as collisions of neutron (or strange) stars or the merge of a binary system of neutron (or strange) stars might generate fragments of SQM whose dimension and velocity spectrum is not easy to guess [2]. In [4] it is proposed the idea that slightly positively charged SQM nuggets engulfed in an electronic atmosphere called nuclearites might account for the DM content in our Universe. Nuclearites would possess a typical velocity of $250 \mathrm{~km} / \mathrm{s}$ near the Earth and density $\rho$, typical of nuclear matter. It is also suggested that such objects - while travelling through the Earth's atmosphere - would deposit part of their energy in the form of luminous radiation and would therefore appear as particularly fast meteors. The DIMS experiment capitalizes on this feature to search for meteor-like events that could be related to the passage of a macro in the atmosphere [5]. It should be noted that the portion of energy that is ultimately released as light heavily depends on the specific model for light emission and on the density of such objects. For example, comparing the brightness of macros of nuclear density and with a mass of $1 \mathrm{~g}$ retrieved using the approaches outlined in [4] and [6] leads to a difference in the visual magnitude of $\Delta M \approx+42$.

\subsection{Interstellar Meteoroids}

The motion of the solar system through the Local Interstellar Cloud (LIC)[7] leads to the presence of interstellar particles, which arrive at the edge of our system at speeds of about $26 \mathrm{~km}$ $\mathrm{s}^{-1}$, reflecting the Sun's velocity relative to the LIC. For interstellar meteoroids that come from other star systems, the corresponding speed is a superposition of their ejection velocity and the star's velocity relative to the Sun. The speed of a meteoroid, measured by a detector at Earth, has an additional component resulting from its fall into the Sun's potential well [8]. Namely, an interstellar meteoroid with an arrival speed $v_{a}$ of e.g. $20 \mathrm{~km} \mathrm{~s}^{-1}$ will have, according to the fundamental relation $v_{H}=\sqrt{42^{2}+v_{a}^{2}}$, a heliocentric speed $v_{H}$ at the Earth's distance of $46.5 \mathrm{~km} \mathrm{~s}^{-1}$, which is about 4.5 $\mathrm{km} \mathrm{s}^{-1}$ above escape velocity from the Sun near Earth. In any case, interstellar meteoroids move in the solar system on hyperbolic orbits with respect to the Sun, and their heliocentric speeds exceed 
the parabolic velocity by several kilometers per second. The measured speeds of particles with hyperbolic heliocentric velocities at the Earth depend on the encounter geometry. Their geocentric velocities may exceed the maximum collisional velocity of two solar system objects, which is 72 $\mathrm{km} \mathrm{s}^{-1}$, but they may also be as low as $12 \mathrm{~km} \mathrm{~s}^{-1}$.

Since the type of the orbit is determined by the velocity of the particle on the orbit around the Sun (for a constant distance $r$ from the Sun), it is strongly influenced by the determination of the resulting heliocentric velocity. This value is derived from the measured speed and influenced by the measured position of the meteor, and it is often burdened by various procedural errors, which depend on the observational method used as well as for the data reduction [10]. It was theoretically shown that a positive error of only $1 \mathrm{~km} \mathrm{~s}^{-1}$ in the pre-atmospheric speed and a change of $1 \mathrm{deg}$ in the radiant position produces hyperbolic orbits in more than one third of the created clones, when applied to a typical perseid-type test particle [9]. Thus, when analysing hyperbolic meteors, with the goal of finding interstellar meteoroids, the influence of errors has to be thoroughly examined, since they may create a spurious population of non-real hyperbolic meteors. Processes in the solar system that may accelerate the particles to hyperbolic orbits are very rare but can be classified and are thus discernible from true interstellar meteoroids [15].

To date, the LIC is the only confirmed source of interstellar particles that were measured by dust detectors, mainly on board the Ulysses and Galileo spacecraft [11],[12]. Whether an interstellar particle reaches the inner part of the solar system, and approaches the Earth's orbit or not, depends on its mass, size, composition and morphology [13],[14]. However, there is a possibility of observing interstellar particles in the Earth's atmosphere as meteors, but their identification assumes high accuracy orbits.

\section{DIMS Experiment}

\subsection{Observation concept}

Observation concept of the DIMS experiment is shown in Fig.1. We are going to observe light emitted from meteoroids and nuclearites in the atmosphere. There is a significant difference in the emission mechanism between them. Therefore, they can be easily distinguished from each other by the speed of emission and the height of the tracks. The meteoroids evaporate when they enter the atmosphere and emit light due to the ionized gas. On the other hand, the nuclearites quasi-elastically collide with ambient atoms, causing black-body radiation from an expanding cylindrical thermal shock. Since the ordinary meteors are bound within the solar system, their heliocentric velocity is less than $42 \mathrm{~km} / \mathrm{s}$. In case of interstellar meteoroids their heliocentric velocity is larger than $42 \mathrm{~km} / \mathrm{s}$. In con-

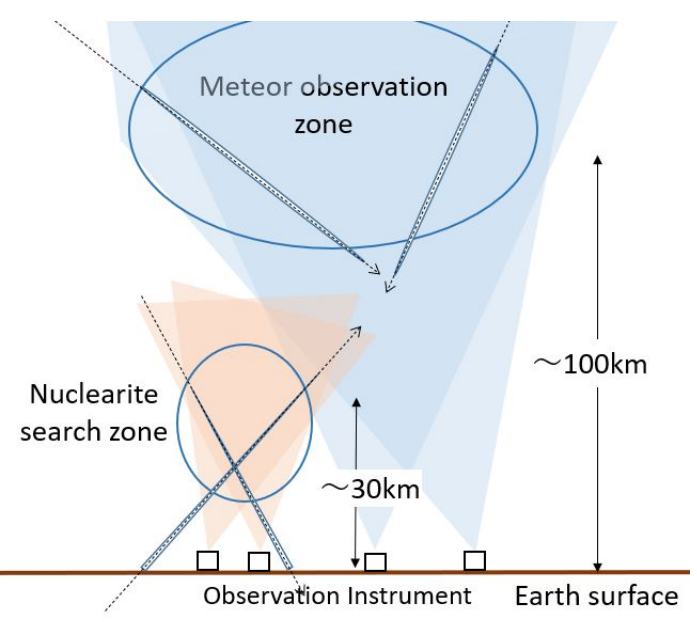

Figure 1: Observation concept of the DIMS experiment. trast to these cases, the macros such as the nuclearites are bound in our galaxy, and their typical 
velocity is about $250 \mathrm{~km} / \mathrm{s}$ in the galactic frame. Also, meteoroids have an emission altitude of about $100 \mathrm{~km}$, whereas the nuclearites have an emission altitude of about $30 \mathrm{~km}$ or less for the masses we are interested in. Finally, the most significant difference between the two is that the meteoroids do not penetrate the Earth, while nuclearites with masses greater than $0.1 \mathrm{~g}$ have the potential to penetrate the Earth. As a result, the nuclearites may make upward trajectories from the Earth's surface to the sky.

\subsection{Detector concept}

To observe such faint, fast moving event tracks, we use multiple high-sensitivity camera modules. Each module consists of a Canon ME20F-SH monochrome camera, 35mm f/1.4 lens, a PC and many other parts. These are installed in a stainless steel box with an acrylic dome. Figure 2 shows a picture of the camera module. Though the maximum ISO sensitivity of the camera is 4 million equivalent, we set the ISO sensitivity at 204,000 for the actual observation at Utah in 2019 because the noise level increases more as the sensitivity increases. We operate the camera with video mode at a high frame rate to obtain the velocity of each event. Accurate velocity and trajectory of each event are able to be obtained by a stereo observation using multiple camera modules. Each

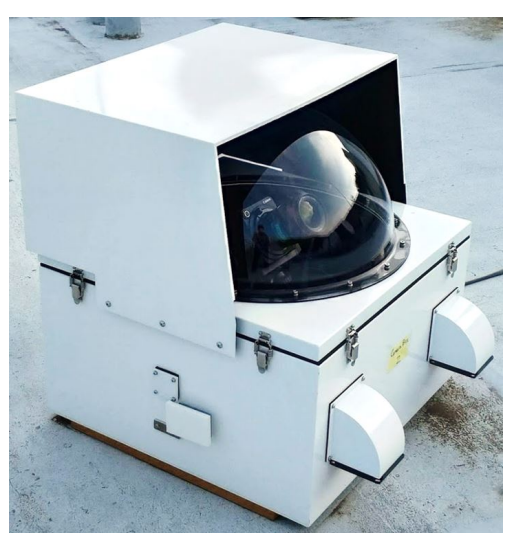

Figure 2: DIMS camera module. camera module has a wild Field of View (FOV) of $56.2^{\circ} \times 33.4^{\circ}$.

\subsection{Camera system}

A block diagram of the camera system in each camera module is shown in Fig. 3. Video images are taken by the camera at a frame rate of $29.97 \mathrm{fps}$ or $59.94 \mathrm{fps}$ with a pixel resolution of $1920 \times 1080$ and their HDMI signals are sent to a PC through an image capture device. Tracks in each image are triggered by a software named UFOCapture [16]. The video images of the triggered events are stored in an SSD device in the PC with avi uncom-

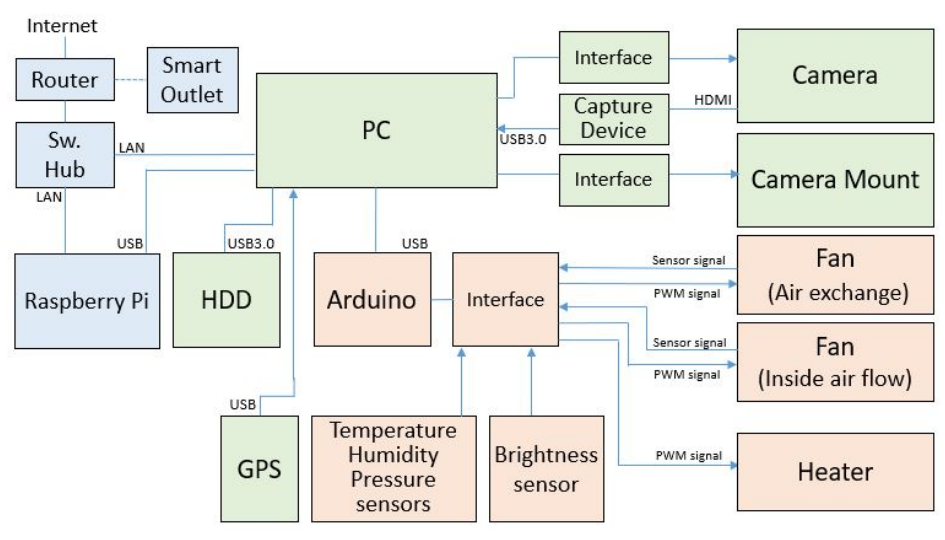

Figure 3: DIMS camera system. pressed format. Still images of the events are also stored with a jpg format. An Arduino microcontroller reads temperatures, humidities, atmospheric pressures inside and outside the camera box and controls 2 fans and a heater to stabilize the temperature and humidity inside the box. Details of the environmental monitor and control system are described elsewhere in these conference proceedings [17]. The camera sys- 
tems are controlled remotely through the Internet by using TeamViewer remote access and control software.

\subsection{Observation site}

The observation system of the DIMS experiment will be deployed at the Telescope Array (TA) high-energy cosmic-ray experiment site [18] in Utah, USA (Fig. 4). Four camera systems will be installed at Central Laser Facility (CLF), Black Rock Mesa (BRM) and TARA at the first stage. One more camera system will be installed later. Base line distances between them are roughly 20 $\mathrm{km}$. Two camera systems will be set at TARA site. Since there is no power lines from the electric power company at the CLF site, we developed an electric power self-sufficiency system using solar power generation [17].

\subsection{Observation}

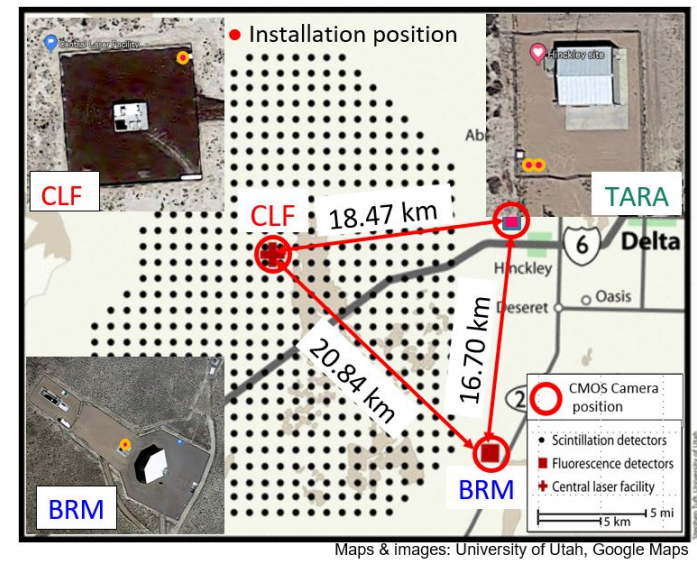

Figure 4: Observation site at TA in Utah. Camera modules will be installed at red marked positions.

Test observations of the DIMS experiment has been performed in Hyogo and Okinawa, Japan and at TA site in Utah from 2017 to 2019 to determine the suitable system and conditions for the experiment [5]. We observed the north centered at Polaris using 2 Canon ME20F-SH monochrome cameras without the camera box at 2 sites shown in Fig. 4 for 4 nights in 2019. An example of composite images of a $6.5 \mathrm{~h}$ observation on September 1st is shown in Fig. 5. We found over 500 meteor events in each

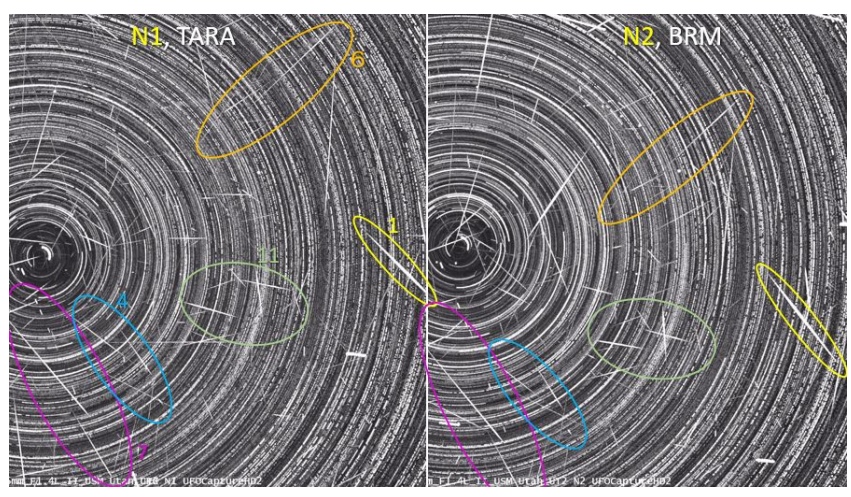

Figure 5: Composite images of meteor events observed in Utah one night in 2019. camera and many simultaneous events on this night.

We have set 3 camera modules at rooftops of 3 universities in Japan from April, 2021 and we have been continuing observation and system development until we move them to Utah.

\section{Analysis}

First, we analyze the observation data with the software called UFOAnalyzerV2 [16] to classify whether they are meteors or not, and obtain parameters such as apparent magnitudes, angular velocities of the meteors and so on. 
We also developed a software to analyze the observation data [19]. Meteor finding algorithms, astrometry/photometry calibration algorithms, triangulation algorithms to get meteor velocity, magnitude etc. are incorporated in the software. We calibrated the cameras by irradiating very uniform light with a $2 \mathrm{~m} \phi$ large integrating sphere at the National Institute of Polar Research in Japan. We can also perform astrometrical and photometrical calibration to the data by using over 1,000 stars with apparent magnitude brighter than $\sim 8$ mag in each event image [19].

\section{Results and Discussion}

Obtained apparent magnitude as a function of angular velocity of the meteors is shown in Fig. 6 for the one night data of Sept. 1st, 2019. Observable limiting-magnitude line for moving objects is drawn in this figure. Expected flux limits for macros and interstellar meteors as a function of their mass can be estimated from this relation.

Distribution of the geocentric meteor velocity was also obtained for the same data. We did not find any events which have velocities faster than $72 \mathrm{~km}$ in this data.

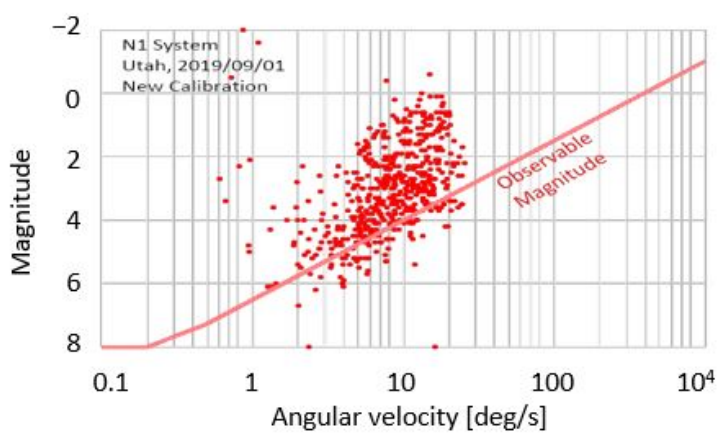

Figure 6: Meteor magnitude vs. angular velocity

Details of the analysis results are also described elsewhere in these conference proceedings [19].

\section{Conclusion}

We have initiated the DIMS experiment project to search for macroscopic dark matter and to study interstellar meteoroids as an international collaboration. Based on preliminary experiments we designed high sensitivity camera modules to deploy at the TA experiment site in Utah. Observation using 3 camera modules in Japan has been already started and is well progressing by using remote control software at present. We will transport them to Utah and start observation there in the very near future.

\section{Acknowledgement}

This work is partially supported by JSPS KAKENHI Grant Number JP19H01910 and the joint research program of the Institute for Cosmic Ray Research (ICRR), the University of Tokyo and by National Science Centre, Poland grant 2020/37/B/ST9/01821. We thanks to members of Telescope Array experiment for their help to achieve the observation, and also Appex corporation and Canon Marketing Japan Inc. for their help related to the high sensitivity CMOS cameras. 


\section{References}

[1] D. M. Jacobs, G. D. Starkman and B. W. Lynn, Monthly Notices of the Royal Astronomical Society 450, pp. 3418-3430 (2015).

[2] Edward Witten, Phys. Rev. D 30, pp. 272-285 (1984).

[3] F. Weber, Progress in Particle and Nuclear Physics 54, pp. 193-288 (2005).

[4] A. De Rújula and S. L. Glashow, Nature 312, 734 (1984).

[5] F. Kajino, I. Ide, R. Ide, Y. Tameda, K. Shinozaki, M. Bertaina, A. Cellino, M.Casolino, T. Ebisuzaki, Y. Takizawa, L. W. Piotrowski, H. Sagawa and J. N. Matthews, Proc. Sci. (ICRC2019) 525, 2019.

[6] J. Sidhu, R. M. Abraham, C. Covault and G. D. Starkman, Journal of Cosmology and Astroparticle Physics 2019, 037 (2019).

[7] Frisch, P.C., Dorschner, J.M., Geiss, J., Greenberg, J.M., Grün, E., Landgraf, M., Hoppe, P., Jones, A.P., Krätschmer, W., Linde, T.J., Morfill, G.E., Reach, W., Slavin, J.D., Svestka, J., Witt, A.N., Zank, G.P., APJ (Acta Pathol. Jpn.) 525, 492-516, 1999.

[8] Hajdukova Jr., M., Sterken, V., Wiegert, P., Cambridge University Press, Cambridge, UK, pp. 235-252, 2019.

[9] Hajdukova Jr., M., Kornos, L., Planet. Space Sci. 190, 104965, 2020.

[10] Hajdukova Jr., M., Sterken, V., Wiegert, P., Planet. Space Sci. 192 (2020) 105060, 2020.

[11] Kruger, H., Strub, P., Grun, E., and Sterken, V. J., The Astrophysical Journal, 812, 139, 2015.

[12] Sterken, V. J., Strub, P., Kruger, H., von Steiger, R., and Frisch, P.,The Astrophysical Journal, $812,141,2015$.

[13] Sterken, V.J., Altobelli, N., Kempf, S., Schwehm, G., Srama, R., Grün, E., A\&A 538, A102, 2012.

[14] Strub, P., Sterken, V.J., Soja, R., Krüger, H., Grün, E., Srama, R., A\&A 621, A54, 2019.

[15] Wiegert, P.A., Icarus 242, 112-121, 2014.

[16] SonotaCo., http://sonotaco.com.

[17] D. Shinto et al., Proc. Sci. (ICRC2021) 502, 2021.

[18] http://www.telescopearray.org/

[19] D. Barghini et al., Proc. Sci. (ICRC2021) 500, 2021. 